\title{
Soft Shadow Maps: Efficient Sampling of Light Source Visibility
}

Lionel Atty $^{1}$, Nicolas Holzschuch ${ }^{1}$, Marc Lapierre ${ }^{2}$, Jean-Marc Hasenfratz ${ }^{1,3}$, Charles Hansen ${ }^{4}$ and François X. Sillion ${ }^{1}$

${ }^{1}$ ARTIS/GRAVIR-IMAG INRIA $\quad{ }^{2}$ MOVI/GRAVIR-IMAG INRIA

${ }^{3}$ Université Pierre Mendès-France $\quad{ }^{4}$ School of Computing, University of Utah
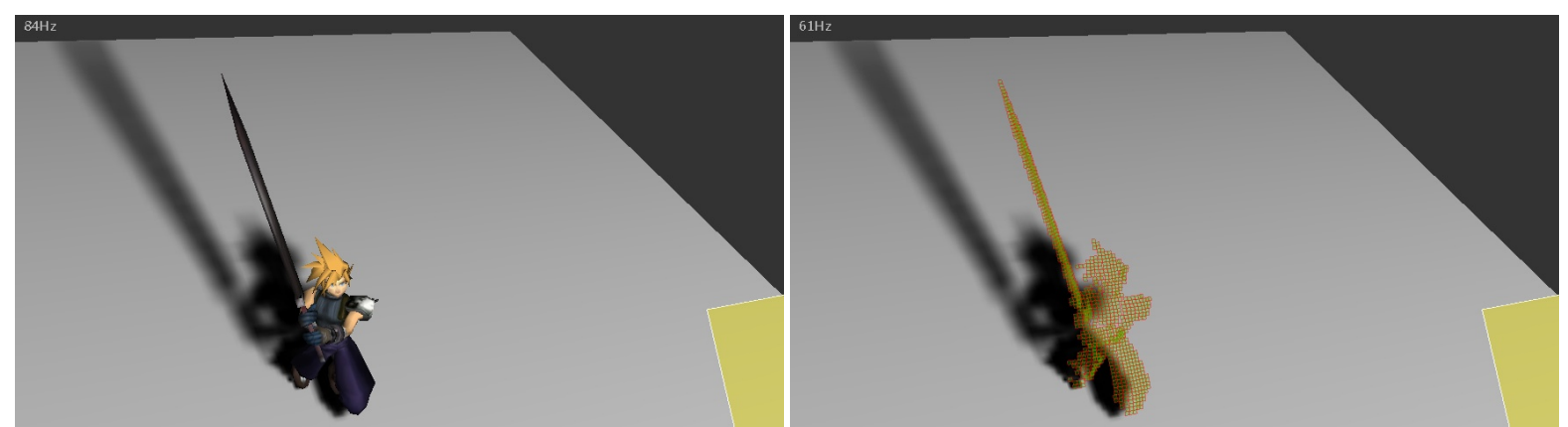

Figure 1: Our algorithm computes soft shadows in real-time (left) by replacing the occluders with a discretized version (right), using information from the shadow map. This scene runs at 84 fps.

\begin{abstract}
Shadows, particularly soft shadows, play an important role in the visual perception of a scene by providing visual cues about the shape and position of objects. Several recent algorithms produce soft shadows at interactive rates, but they do not scale well with the number of polygons in the scene or only compute the outer penumbra. In this paper, we present a new algorithm for computing interactive soft shadows on the GPU. Our new approach provides both inner-and outer-penumbra, and has a very small computational cost, giving interactive frame-rates for models with hundreds of thousands of polygons.

Our technique is based on a sampled image of the occluders, as in shadow map techniques. These shadow samples are used in a novel manner, computing their effect on a second projective shadow texture using fragment programs. In essence, the fraction of the light source area hidden by each sample is accumulated at each texel position of this Soft Shadow Map. We include an extensive study of the approximations caused by our algorithm, as well as its computational costs.
\end{abstract}

Categories and Subject Descriptors (according to ACM CCS): I.3.1 [Computer Graphics]: Graphics processors I.3.7 [Computer Graphics]: Color, shading, shadowing, and texture

\section{Introduction}

Shadows add important visual information to computergenerated images. The perception of spatial relationships between objects can be altered or enhanced simply by modifying the shadow shape, orientation, or position [WFG92, Wan92,KMK97]. Soft shadows, in particular, provide robust contact cues by the hardening of the shadow due to prox- imity resulting in a hard shadow upon contact. The advent of powerful graphics hardware on low-cost computers has led to the emergence of many interactive soft shadow algorithms (for a detailed study of these algorithms, please refer to [HLHS03]).

In this paper, we introduce a novel method based on shadow maps to interactively render soft shadows. Our

(c) The Eurographics Association and Blackwell Publishing 2006. Published by Blackwell Publishing, 9600 Garsington Road, Oxford OX4 2DQ, UK and 350 Main Street, Malden, MA 02148 , USA. 


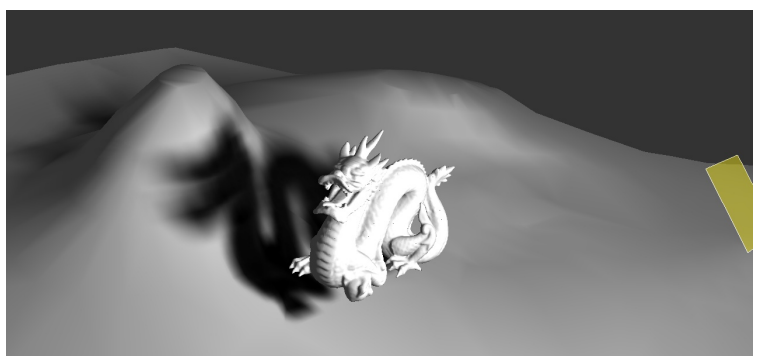

Figure 2: Applying our algorithm (200,000 polygons, occluder map $256 \times 256$, displayed at $32 \mathrm{fps})$.

method interactively computes a projective shadow texture, the Soft Shadow Map, that incorporates soft shadows based on light source visibility from receiver objects (see Fig. 2). This texture is then projected onto the scene to provide interactive soft shadows of dynamic objects and dynamic area light sources.

There are several advantages to our technique when compared to existing interactive soft-shadow algorithms: First, it is not necessary to compute silhouette edges. Second, the algorithm is not fill-bound, unlike methods based on shadow volumes. These properties provide better scaling for occluding geometry than other GPU based soft shadow techniques [WH03, CD03, AAM03]. Third, unlike some other shadow map based soft shadow techniques, our algorithm does not dramatically overestimate the umbra region [WH03, CD03]. Fourth, while other methods have relied on an interpolation from the umbra to the non-shadowed region to approximate the penumbra for soft shadows [AHT04, WH03, CD03, BS02], our method computes the visibility of an area light source for receivers in the penumbra regions.

Our algorithm also has some limitations when compared to existing algorithms. First, our algorithm splits scene geometry into occluders and receivers and self shadowing is not accounted for. Also, since our algorithm uses shadow maps to approximate occluder geometry, it inherits the well known issues with aliasing from shadow map techniques. For large area light sources, the soft shadows tend to blur the artifacts but for smaller area light sources, such aliasing is apparent.

We acknowledge that these limitations are important, and they may prevent the use of our algorithm in some cases. However, there are many applications such as video games or immersive environments where the advantages of our algorithm (a very fast framerate, and a convincing soft shadow) outweigh its limitations. We also think that this new algorithm could be the start of promising new research.

In the following section, we review previous work on interactive computation of soft shadows. In Section 3, we present the basis of our algorithm, and in the following section, we provide implementation details. In the next two sec- tions, we conduct an extensive analysis of our algorithm; first, in Section 5, we study the approximations in our soft shadows, then in Section 6 we study the rendering times of our algorithm. Both studies are done first from a theoretical point of view, then experimentally. Finally, in Section 7, we conclude and expose possible future directions for research.

\section{Previous Work}

Researchers have investigated shadow algorithms for computer-generated images for nearly three decades. The reader is referred to a recent state-of-the art report by Hasenfratz et al. [HLHS03], the overview by Woo et al. [WPF90] and the book by Akenine-Möller and Haines [AMH02].

The two most common methods for interactively producing shadows are shadow maps [Wil78] and shadow volumes [Cro77]. Both of these techniques have been extended for soft shadows. In the case of shadow volumes, Assarsson and Akenine-Möller [AAM03] used penumbra wedges in a technique based on shadow volumes to produce soft shadows. Their method depends on locating silhouette edges to form the penumbra wedges. While providing good soft shadows without an overestimate of the umbra, the algorithm is fill-limited, particularly when zoomed in on a soft shadow region. Since it is necessary to compute the silhouette edges at every frame, the algorithm also suffers from scalability issues when rendering occluders with large numbers of polygons.

The fill-rate limitation is a well known limitation of shadow-volume based algorithms. Recent publications [CD04, LWGM04] have focused on limiting the fillrate for shadow-volume algorithms, thus removing this limitation.

On shadow maps, Chan and Durand [CD03] and Wyman and Hansen [WH03] both employed a technique which uses the standard shadow map method for the umbra region and builds a map containing an approximate penumbra region that can be used at run-time to give the appearance, including hard shadows at contact, of soft shadows. While these methods provide interactive rendering, both only compute the outer-penumbra, the part of the penumbra that is outside the hard shadow. In effect, they are overestimating the umbra region, resulting in the incorrect appearance of soft shadows in the case of large area light sources. These methods also depend on computing the silhouette edges in object space for each frame; this requirement limits the scalability for occluders with large numbers of polygons.

Arvo et al. [AHT04] used an image-space flood-fill method to produce approximate soft shadows. Their algorithm is image-based, like ours, but works on a detection of shadow boundary pixels, followed by several passes to replace the boundary by a soft shadow, gradually extending the soft shadow at each pass. The main drawback of their method is that the number of passes required is proportional 
to the extent of the penumbra region, and the rendering time is proportional to the number of shadow-filling passes.

Guennebaud et al. [GBP06] also used the back projection of each pixel in the shadow map to compute the soft shadow. Their method was developed independently of ours, yet is very similar. The main differences between the two methods lie in the order of the computations: we compute the soft shadow in shadow map space, while they compute the soft shadow in screen space, requiring a search in the shadow map.

Brabec and Seidel [BS02] and Kirsch and Doellner [KD03] use a shadow map to compute soft shadows, by searching at each pixel of the shadow map for the nearest boundary pixel, then interpolating between illumination and shadow as a function of the distance between this pixel and the boundary pixel and the distances between the light source, the occluder and the receiver. Their algorithm requires scanning the shadow map to look for boundary pixels, a potentially costly step; in practical implementations they limit the search radius, thus limiting the actual size of the penumbra region.

Soler and Sillion [SS98] compute a soft shadow map as the convolution of two images representing the source and blocker. Their technique is only accurate for planar and parallel objects, although it can be extended using an object hierarchy. Our technique can be seen as an extension of this approach, where the convolution is computed for each sample of an occlusion map, and the results are then combined.

Finally, McCool [McC00] presented an algorithm merging shadow volume and shadow map algorithms by detecting silhouette pixels in the shadow map and computing a shadow volume based on these pixels. Our algorithm is similar in that we are computing a shadow volume for each pixel in the shadow map. However, we never display this shadow volume, thus avoiding fill-rate issues.

\section{Algorithm}

\subsection{Presentation of the algorithm}

Our algorithm assumes a rectangular light source and starts by separating potential occluders (such as moving characters) from potential receivers (such as the background in a scene) (Fig. 3(a)). We will compute the soft shadows only from the occluders onto the receivers.

Our algorithm computes a Soft Shadow Map, (SSM), for each light source: a texture containing the texelwise percentage of occlusion from the light source. This soft shadow map is then projected onto the scene from the position of the light source, to give soft shadows (see Fig. 2).

Our algorithm is an extension of the shadow map algorithm: we start by computing depth buffers of the scene. Unlike the standard shadow map method, we will need two

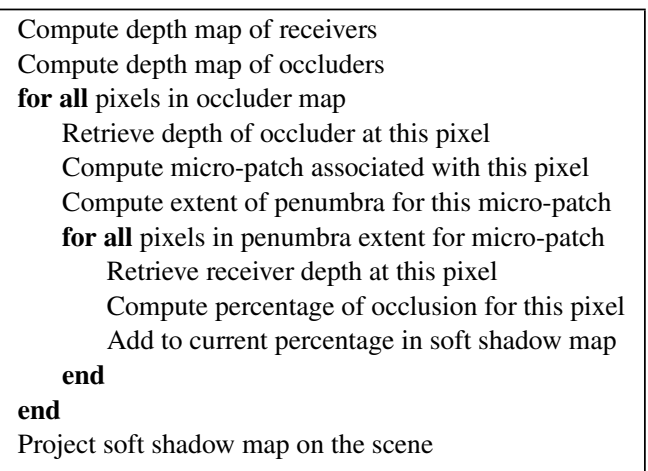

Figure 4: Our algorithm

depth buffers: one for the occluders (the occluder map) and the other for the receivers.

The occluder map depth buffer is used to discretize the set of occluders (see Fig. 3(b)): each pixel in this occluder map is converted into a micro-patch that covers the same image area but is is located in a plane parallel to the light source, at a distance corresponding to the pixel depth. Pixels that are close to the light source are converted into small rectangles and pixels that are far from the light source are converted into larger rectangles. At the end of this step, we have a discrete representation of the occluders.

The receiver map depth buffer will be used to provide the receiver depth, as our algorithm uses the distance between light source and receiver to compute the soft shadow values.

We compute the soft shadow of each of the micro-patches constituting the discrete representation of the occluders (see Fig. 3(c)), and sum them into the soft shadow map (SSM) (see Fig. 3(d)). This step would be potentially costly, but we achieve it in a reasonable amount of time with two key points: 1) the micro-patches are parallel to the light source, so computing their penumbra extent and their percentage of occlusion only requires a small number of operations, and 2) these operations are computed on the graphics card, exploiting the parallelism of the GPU engine. The percentage of occlusion from each micro-patch takes into account the relative distances between the occluders, the receiver and the light source. Our algorithm introduces several approximations on the actual soft shadow. These approximations will be discussed in Section 5 .

The pseudo-code for our algorithm is given in Fig. 4. In the following subsections, we will review in detail the individual steps of the algorithm: discretizing the occluders (Section 3.2), computing the penumbra extent for each micro-patch (Section 3.3) and computing the percentage of occlusion for each pixel in the Soft Shadow Map (Section 3.4). Specific implementation details will be given in Section 4. 


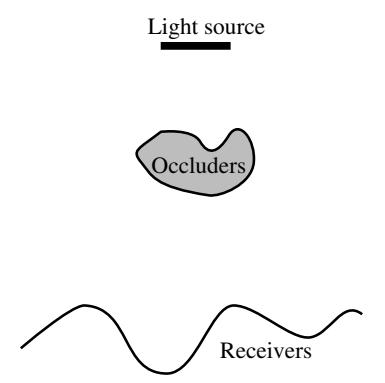

(a) Scene view

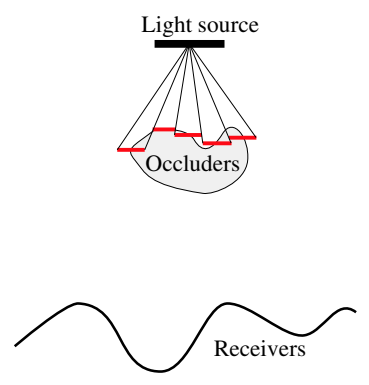

(b) Discretizing occluders

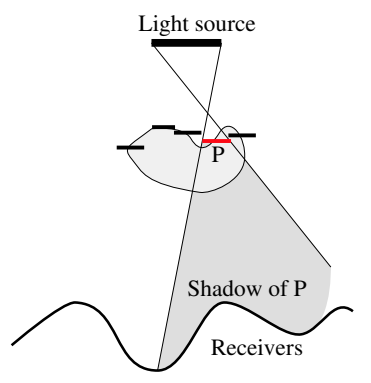

(c) Soft shadows from one micro-patch

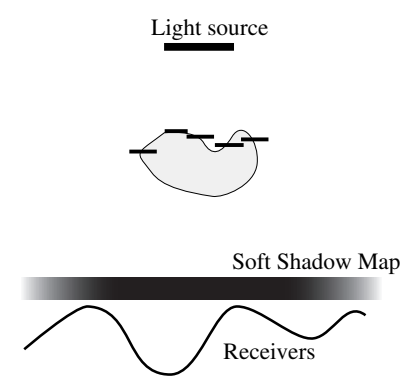

(d) Summing the soft shadows

Figure 3: The main steps of our algorithm

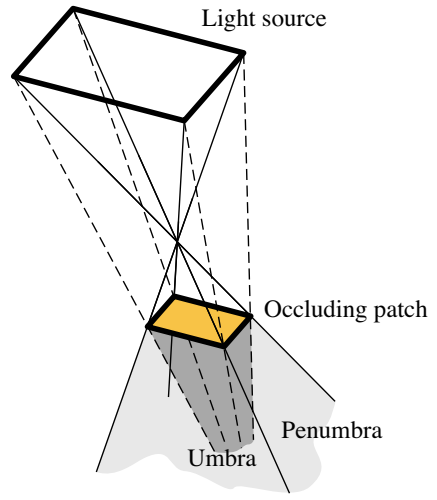

Figure 5: The penumbra extent of a micro-patch is a rectangular pyramid

\subsection{Discretizing the occluders}

The first step in our algorithm is a discretization of the occluders. We compute a depth buffer of the occluders, as seen from the light source, then convert each pixel in this occluder map into the equivalent polygonal micro-patch that lies in a plane parallel to the light source, at the appropriate depth and occupies the same image plane extent (see Fig. 1).

The occluder map is axis-aligned with the rectangular light source and has the same aspect ratio: all micro-patches created in this step are also axis-aligned with the light source and have the same aspect ratio.

\subsection{Computing penumbra extents}

Each micro-patch in the discretized occluder is potentially blocking some light between the light source and some portion of the receiver. To reduce the amount of computations, we compute the penumbra extent of the micro-patches, and we only compute occlusion values inside these extents.

Since the micro-patches are parallel, axis-aligned with the

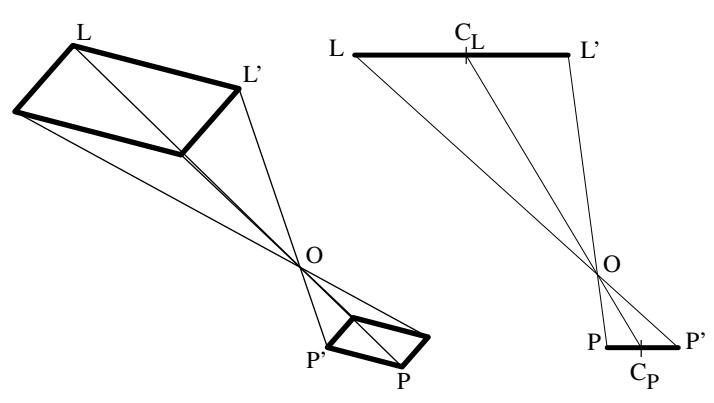

(a)

(b)

Figure 6: Finding the apex of the pyramid is reduced to a $2 D$ problem

light source and have the same aspect ratio, the penumbra extent of each micro-patch is a rectangular pyramid (Fig. 5). Finding the penumbra extent of the light source is equivalent to finding the apex $O$ of the pyramid (Fig. 6(a)). This reduces to a $2 \mathrm{D}$ problem, considering parallel edges $\left(L L^{\prime}\right)$ and $\left(P P^{\prime}\right)$ on both polygons (Fig. $6(\mathrm{~b}))$. Since $\left(L L^{\prime}\right)$ and $\left(P P^{\prime}\right)$ are parallel lines, we have:

$$
\frac{O L}{O P}=\frac{O L^{\prime}}{O P^{\prime}}=\frac{L L^{\prime}}{P P^{\prime}}
$$

This ratio is the same if we consider the center of each line segment:

$$
\frac{O C_{L}}{O C_{P}}=\frac{L L^{\prime}}{P P^{\prime}}
$$

Since the micro-patch and the light source have the same aspect ratio, the ratio $r=\frac{L L^{\prime}}{P P^{\prime}}$ is the same for both sides of the micro-patch (thus, the penumbra extent of the micro-patch is indeed a pyramid).

We find the apex of the pyramid by applying a scaling to the center of the micro-patch $\left(C_{P}\right)$, with respect to the center 


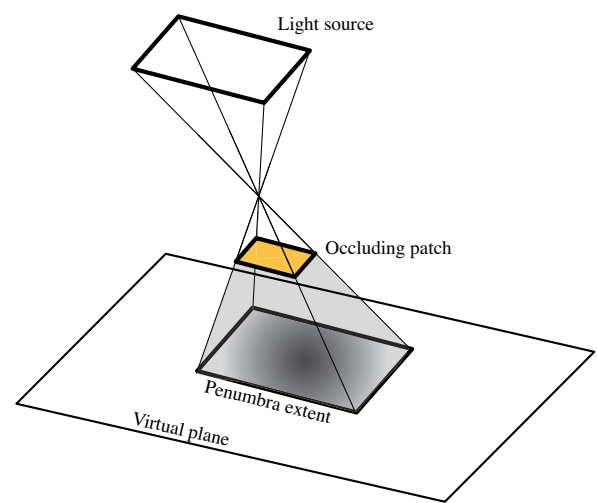

Figure 7: The intersection between the pyramid and the virtual plane is an axis-aligned rectangle

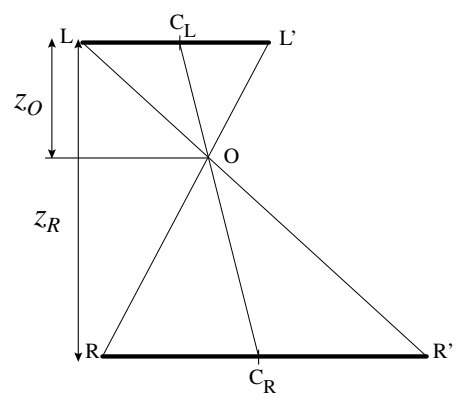

Figure 8: Computing the position and extent of the penumbra rectangle for each micro-patch.

of the light source $\left(C_{L}\right)$ :

$$
\overrightarrow{C_{L} O}=\frac{r}{1+r} \overrightarrow{C_{L} C_{P}}
$$

where $r$ is again the ratio $r=\frac{L L^{\prime}}{P P^{\prime}}$.

We now use this pyramid to compute occlusion in the soft shadow map (see Fig. 7). We use a virtual plane, parallel to the light source, to represent this map (which will be projected onto the scene). The intersection of the penumbra pyramid with this virtual plane is an axis-aligned rectangle. We only have to compute the percentage of occlusion inside this rectangle.

Computing the position and size of the penumbra rectangle uses the same formulas as for computing the apex of the pyramid (see Fig. 8):

$$
\begin{aligned}
\overrightarrow{C_{L} C_{R}} & =\frac{z_{R}}{z_{O}} \overrightarrow{C_{L} O} \\
R R^{\prime} & =L L^{\prime} \frac{z_{R}-z_{O}}{z_{O}}
\end{aligned}
$$

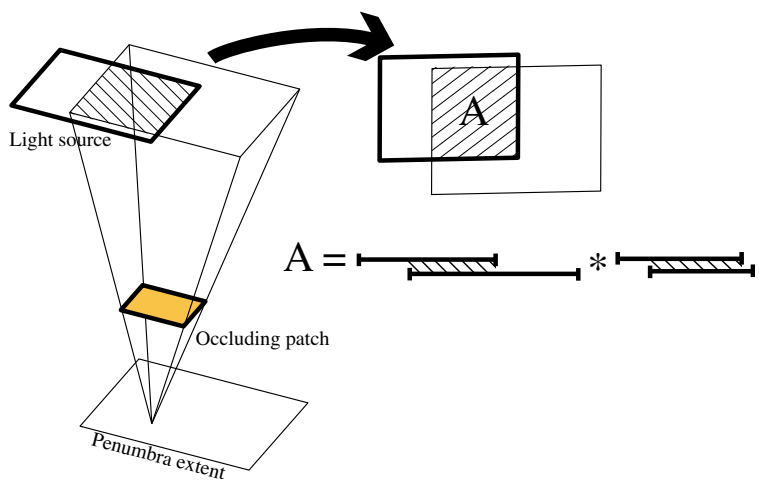

Figure 9: We reproject the occluding micro-patch onto the light source and compute the percentage of occlusion.

\subsection{Computing the soft shadow map}

For all the pixels of the SSM lying inside this penumbra extent, we compute the percentage of the light source that is occluded by this micro-patch. This percentage of occlusion depends on the relative positions of the light source, the occluders and the receivers. To compute it, for each pixel on the receiver inside this extent, we project the occluding microfacet back onto the light source [DF94] (Fig. 9). The result of this projection is an axis-aligned rectangle; we need to compute the intersection between this rectangle and the light source.

Computing this intersection is equivalent to computing the two intersections between the respective intervals on both axes. This part of the computation is done on the GPU, using a fragment program: the penumbra extent is converted into an axis-aligned quad, which we draw in a float buffer. For each pixel inside this quad, the fragment program computes the percentage of occlusion. These percentages are summed using the blending capability of the graphics card (see Section 4.2).

\subsection{Two-sided soft-shadow maps}

As with many other soft shadow computation algorithms [HLHS03], our algorithm exhibits artifacts because we are computing soft shadows using a single view of the occluder. Shadow effects linked to parts of the occluder that are not directly visible from the light source are not visible. In Fig. 10(a), our algorithm only computes the soft shadow for the front part of the occluder, because the back part of the occluder does not appear in the occluder map. This limitation is frequent in real-time soft-shadow algorithms [HLHS03].

For our algorithm, we have devised an extension that solves this limitation: we compute two occluder maps. In the first, we discretize the closest, front-facing faces of the occluders (see Fig. 10(b)). In the second, we discretize the furthest, back-facing faces of the occluders (see Fig. 10(c)). 


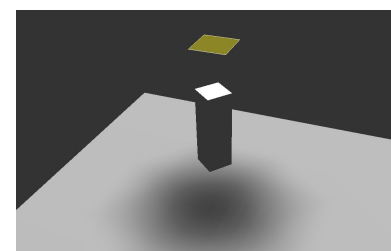

(a) Original algorithm
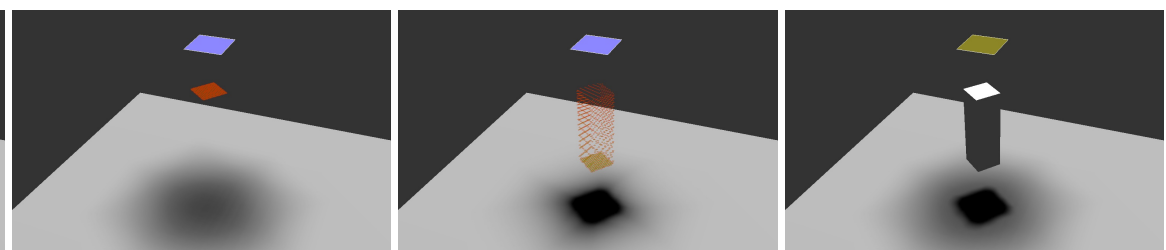

(b) Closest, front faces of the (c) Furthest, back faces of the (d) Combining the two soft occluder discretized with their occluder discretized with their shadow maps shadow shadow

Figure 10: The original algorithm fails for some geometry. The two-pass method gives the correct shadow.

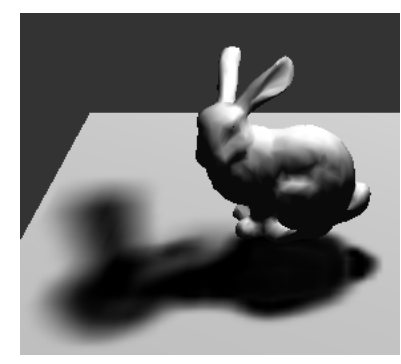

(a) One pass (148 fps)

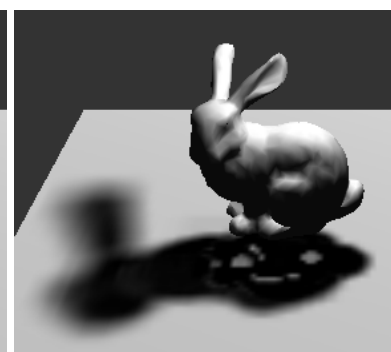

(b) One pass with bottom patches (142 fps)

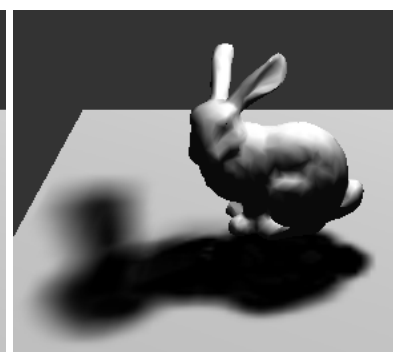

(c) Two passes (84 fps)

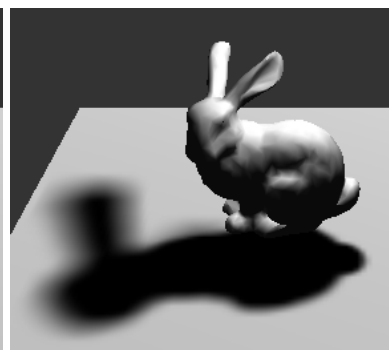

(d) Ground truth

Figure 11: Two-pass shadow computations enhance precision.

We then compute a soft shadow map for each occluder map, and merge them, using the maximum of each occluder map. The resulting occlusion map has eliminated most artifacts (Fig. 10(d) and 11). Empirically, the cost of the twopass algorithm is between 1.6 and 1.8 times the cost of the one-pass algorithm. Depending on the size of a model and the quality requirements of a given application, the second pass may be worth this extra cost. For example, for an animated model of less than 100,000 polygons, the one-pass algorithm renders at approximately $60 \mathrm{fps}$. Adding the second pass drops the framerate to $35 \mathrm{fps}$ - which is still interactive.

\section{Implementation details}

\subsection{Repartition between CPU and GPU}

Our algorithm (see Fig. 4) starts by rendering two depth maps, one for the occluders and one for the receivers; these depth maps are both computed by the GPU. Then, in order to generate the penumbra extents for the micro-patches, the occluders depth map is transferred back to the CPU.

On the CPU, we generate the penumbra extents for the micro-patch associated to each non-empty pixel of the occluders depth map. We then render these penumbra extents, and for each pixel, we execute a small fragment program to compute the percentage of occlusion. Computing the per- centage of occlusion at each pixel of the soft shadow map is done on the GPU (see section 4.2).

These contributions from each micro-patch are added together; we use for this the blending ability of the GPU: occlusion percentages are rendered into a floating-point buffer with blending enabled, thus the percentage values for each micro-patch are automatically added to the previously computed percentage values.

\subsection{Computing the intersection}

For each pixel of the SSM lying inside the penumbra extent of a micro-patch, we compute the percentage of the light source that is occluded by this micro-patch, by projecting the occluding micro-patch back onto the light source (see Fig. 9). We have to compute the intersection of two axisaligned rectangles, which is the product of the two intersections between the respective intervals on both axes.

We have therefore reduced our intersection problem from a $2 \mathrm{D}$ problem to two separate $1 \mathrm{D}$ problems. To further optimize the computations, we use the SAT instructions in the fragment program assembly language: without loss of generality, we can convert the rectangle corresponding to the light source to $[0,1] \times[0,1]$. Each interval intersection becomes the intersection between one $[a, b]$ interval and $[0,1]$. Exploiting the SAT instruction and swizzling, computing the area of the intersection between the projection of the oc- 
cluder $[a, b] \times[c, d]$ and the light source $[0,1] \times[0,1]$ only requires three instructions:

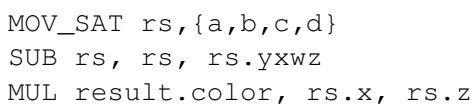

Computing the $[a, b] \times[c, d]$ intervals requires projecting the micro-patch onto the light source and scaling the projection. This uses 8 other instructions: 6 basic operations (ADD, MUL, SUB), one reciprocal (RCP) and one texture lookup to get the depth of the receiver. The total length of our fragment program is therefore 11 instructions, including one texture lookup.

\subsection{Possible improvements}

As it stands, our algorithm makes a very light use of GPU resources: we only execute a very small fragment program, once for each pixel covered by the penumbra extent, and we exploit the blending ability for floating point buffers.

The main bottleneck of our algorithm is that the penumbra extents have to be computed on the CPU. This requires transfering the occluders depth map to the CPU, and looping over the pixels of the occluders depth map on the CPU. It should be possible to remove this step by using the renderto-vertex- buffer function: instead of rendering the occluders depth map, we would directly render the penumbra extents for each micro-patch into a vertex buffer. This vertex buffer would be rendered in a second pass, generating the soft shadow map.

\section{Error Analysis and comparison}

In this section, we analyze our algorithm, its accuracy and how it compares with the exact soft-shadows. We first study potential sources of error from a theoretical point of view, in Section 5.1, then we conduct an experimental analysis, comparing the soft shadows produced with exact soft shadows, in Section 5.2.

\subsection{Theoretical analysis}

Our algorithm replaces the occluder with a discretized version. This discretization ensures interactive framerates, but it can also be a source of inaccuracies. From a given point on the receiver, we are separately estimating occlusion from several micro-patches, and adding these occlusion values together. We have identified three potential sources of error in our algorithm:

- We are only computing the shadow of the discretized occluder, not the shadow of the actual occluder. This source of error will be analyzed in Section 5.1.1.

- The reprojections of the micro-patches on the light source may overlap or be disjoined. This cause of error will be analyzed in Section 5.1.2.
- We are adding many small values (the occlusion from each micro-patch) to form a large value (the occlusion from the entire occluder). If the micro-patches are too small, we run into numerical accuracy issues, especially with floating-point numbers expressed on 16 bits. This cause of error will be analyzed in Section 5.1.3.

\subsubsection{Discretization error}

Our algorithm computes the shadow of the discretized occluder, not the shadow of the actual occluder. The discretized occluder corresponds to the part of the occluder that is visible from the camera used to compute the depth buffers, usually the center of the light source. Although we reproject each micro-patch of the discretized occluder onto the area light source, we are missing the parts of the occluder that are not visible from the shadow map camera but are still visible from some points of the area light source. This is a limitation that is frequent in real-time soft shadow algorithms [HLHS03], especially algorithms relying on the silhouette of the occluder as computed from a single point [WH03, CD03, AAM03].

We also use a discrete representation based on the shadow map, not a continuous representation of the occluder. For each pixel of the shadow map, we are potentially overestimating or underestimating the actual occluder by at most half a pixel.

If the occluder has one or more edges aligned with the edges of the shadow map, these discretization errors are of the same sign over the edge, and add themselves; the worst case scenario is a square aligned with the axis of the shadow map.

For more practical occluders the discretization errors on neighboring micro-patches compensate: some of the micropatches overestimate the occluder while others underestimate it.

\subsubsection{Overlapping reprojections}

At any given point on the receiver, the parts of the light source that are occluded by two neighboring micro-patches should be joined exactly for our algorithm to compute the exact percentage of occlusion on the light source. This is typically not the case, and these parts may overlap or there may be a gap between them (Fig. 12). The amount of overlap (or gap) between the occluded parts of the light source depends on the relative positions of the light source, the occluding micro-patches and the receiver

If we consider the 2D equivalent of this problem (Fig. 13), with two patches separated by $\delta h$ and at a distance $z_{O}$ from the light source, with the receiver being at a distance $z_{R}$ from the light source, there is a point $P_{0}$ on the receiver where there is no overlap between the occluded parts. As we move away from this point, the overlap increases. For a point at a 


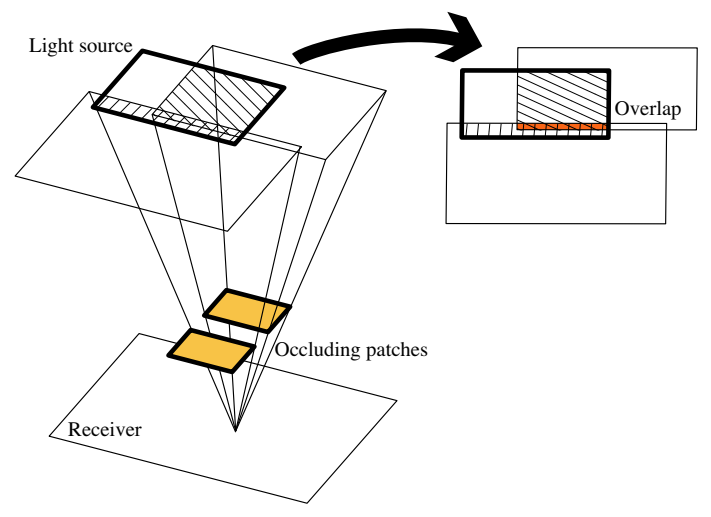

Figure 12: The reprojection of two neighboring micropatches may overlap.

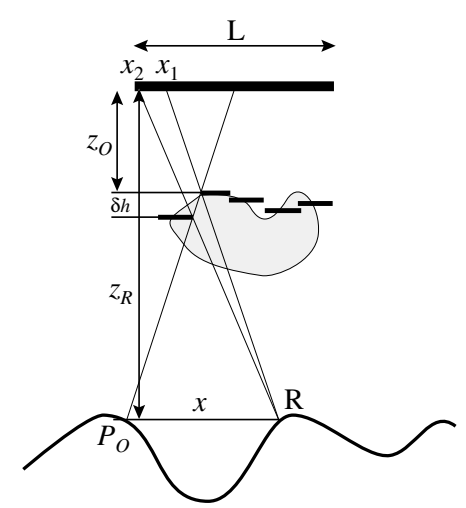

Figure 13: Computing the extent of overlap or gap between two neighboring micro-patches.

distance $x$ from $P_{0}$, the boundaries of the occluding micropatches project at abscissa $x_{1}$ and $x_{2}$; as the occluding micropatches and the light source lie in parallel planes, we have:

$$
\begin{aligned}
\frac{x_{1}}{x} & =\frac{z_{O}}{z_{R}-z_{O}} \\
\frac{x_{2}}{x} & =\frac{z_{O}+\delta h}{z_{R}-z_{O}-\delta h}
\end{aligned}
$$

The amount of overlap is therefore:

$$
\begin{aligned}
x_{2}-x_{1} & =x\left(\frac{z_{O}}{z_{R}-z_{O}}-\frac{z_{O}+\delta h}{z_{R}-z_{O}-\delta h}\right) \\
& =-x \frac{z_{R} \delta h}{\left(z_{R}-z_{O}\right)\left(z_{R}-z_{O}-\delta h\right)}
\end{aligned}
$$

$x$ itself is limited, since the occlusion area must fall inside the light source:

$$
|x|<\frac{L}{2} \frac{z_{R}-z_{O}}{z_{O}}
$$

The amount of overlap is therefore limited by:

$$
\left|x_{2}-x_{1}\right|<\frac{L}{2} \frac{z_{R} \delta h}{z_{O}\left(z_{R}-z_{O}-\delta h\right)}
$$

Equation 3 represents the error our algorithm makes for each pair of micro-patches. The overall error of our algorithm is the sum of the modulus of all these errors, for all the micro-patches projecting on the light source at a given point. This is a conservative estimate, as usually some patches overlap while others present gaps; the actual sum of the occlusion values from all the micro-patches is closer to the real value than what our estimation tells (see Section 5.2).

The theoretical error caused by our algorithm depends on several factors:

Size of the light source: The maximum amount of overlap (Eq. 3) depends directly on the size of the light source. The larger the light source, the larger the error. Our practical experiments confirm this.

Distance between micro-patches: The maximum amount of overlap (Eq. 3) also depends linearly on $\delta h$, the distance in $z$ between neighboring micro-patches. Since $\delta h$ depends on the discretization of the occluder, the error introduced by our algorithm is related to the resolution of the bitmap: the smaller the resolution of the bitmap, the larger the error. Our practical experiments confirm this, but there is a maximum resolution after which the error does not decrease.

Note that this source of error is related to the effective resolution of the bitmap, that is the number of pixels used for discretizing the occluder. If the occluder occupies only a small portion of the bitmap, the effective resolution of the bitmap is much smaller than its actual resolution. Fortunately, the cost of the algorithm is also related to the effective resolution of the bitmap.

Distance to the light source/the receiver: If the occluder touches either the light source or the receiver, the amount of overlap (Eq. 3) goes toward infinity. When the occluder is touching the receiver, the area where the overlap occurs (as defined by equation 2) goes towards 0 , thus the error does not appear. When the occluder is touching the receiver, the actual effect depends on the shape of the occluder. In some cases, overlaps and gaps can compensate, resulting in an acceptable shadow.

\subsubsection{Floating-point blending accuracy}

Our algorithm adds together many small scale occlusion values - the occlusion from each micro-patch - to compute a large scale occlusion value - the occlusion from the complete occluder. This addition is done with the blending ability of the GPU, using blending of floating-point buffers. At the time of writing, blending is only available in hardware for 16-bits floating-point buffers. As a result, we sometimes encounter problems of numerical accuracy. 

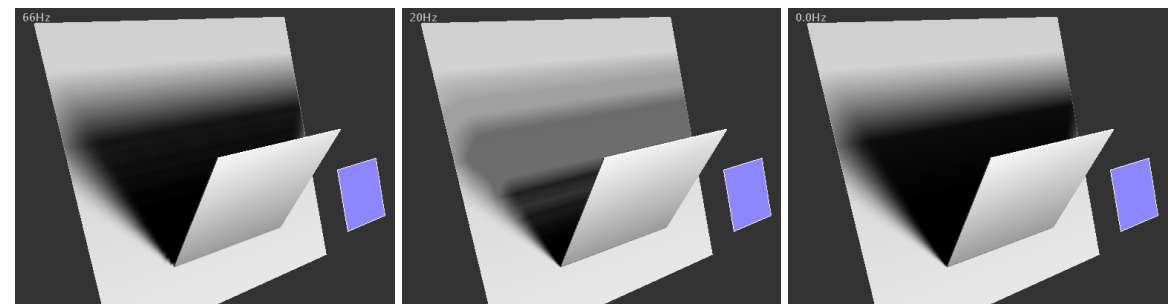
(a) $128^{2}$
$(66 \mathrm{~Hz})$
(20 $\mathrm{Hz})$ pixels, FP16 blending (c)
$512^{2}$ pixels, FP32 blending (CPU)

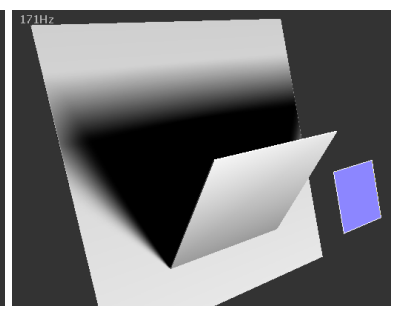

(d) Ground truth (CPU)

Figure 14: Blending with FP16 numbers: if the resolution of the shadow map is too high, numerical issues appear, resulting in wrong shadows. Using higher accuracy for blending removes this issue (here, FP32 blending was done on the CPU).

Figure 14 shows an example of these problems. Unconventionally, increasing the resolution of the shadow map makes these problems more likely to appear (for a complete study of floating-point blending accuracy, see appendix A). The best workaround is therefore to use relatively low resolution for the occluder map, such as $128 \times 128$ or $256 \times 256$. While this may seem a low resolution compared to other shadow map algorithms, our shadow map is focused on the moving occluder (such as a character), not on the entire scene, so $128 \times 128$ pixels is usually enough resolution.

We see this is only as a temporary issue that will disappear as soon as hardware FP32 blending becomes available on graphics cards.

\subsection{Comparison with ground truth}

We ran several tests to experimentally compare the shadows produced by our algorithm with the actual shadows. The reference values were computed using occlusion queries, giving an accurate estimation of the real occlusion of the light source. In this section, we review the practical differences we observed.

\subsubsection{Experimentation method}

For each image, we computed an error metric as thus: for each pixel in the soft shadow map, we compute the actual occlusion value (using occlusion queries), and the difference with the occlusion value computed using our algorithm. We summed the modulus of the differences, then divided the result by the total number of pixels lying either in the shadow or in the penumbra, averaging the error over the actual soft shadow. We used the number of pixels that are either in shadow or in penumbra and not the total number of pixels in the occluders depth map because the soft shadow can occupy only a small part of the depth map. Dividing by the total number of pixels in the depth map would have underestimated the error.

We have used 3 different scenes (a square plane parallel to the light source, a Buddha model and a Bunny model). These scenes exhibit several interesting features. The Buddha and Bunny are complex models, with folds and creases. The Bunny also has important self-occlusion, and in our scene it is in contact with the ground, providing information on the behavior of our algorithm in that case. The square plane is an illustration of the special case of occluders aligned with the axes of the occluders depth map.

We have tested both the one-pass and the two-pass versions of our algorithm. We selected four separate parameters: the size of the light source, the resolution of the shadow map and moving the occluder, either vertically from the receiver to the light source or laterally with respect to the light source. For each parameter, we plot the variation of the error introduced by our algorithm as a function of the parameter and analyze the results.

\subsubsection{Visual comparison with ground truth}

Fig. 16 shows a side by side comparison of our algorithm with ground truth. Even though there are slight differences with ground truth, our algorithm exhibits the proper behavior for soft shadows: sharp shadows at places where the object is close to the ground, a large penumbra zone where the object is further away from the receiver. Our algorithm visibly computes both the inner and the outer penumbra of the object.

Looking at the picture of the differences (Fig. 16(d) and $16(\mathrm{~g})$ ) between the shadow values computed by our algorithm and the ground truth values, it appears that the differences lie mostly on the silhouette: since our algorithm only computes the soft shadow of the discretized object, as seen from the center of the light source. The actual shape of the soft shadow depends on subtle effects happening at the boundary of the silhouette.

\subsubsection{Size of the buffer}

Figure 17 shows the average difference between the occlusion values computed with our algorithm and the actual occlusion values for our three test scenes, when changing the resolution of the shadow map. In these figures, the abscissa 


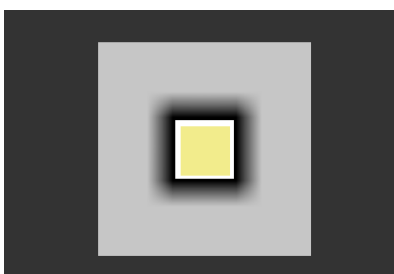

(a) Square plane

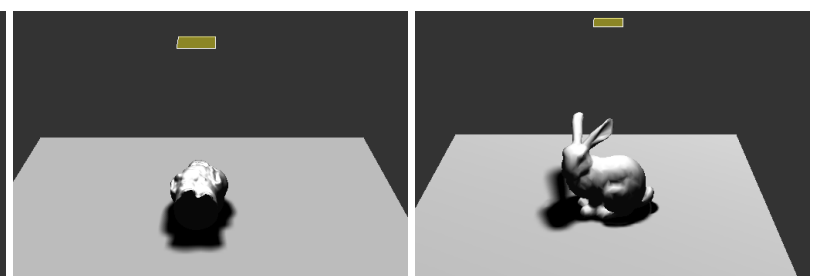

(b) Buddha (c) Bunny

Figure 15: The test scenes we have used

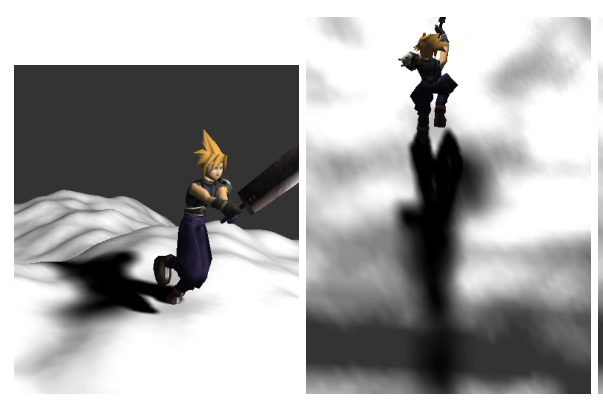

(a) Scene view (b) Our algorithm

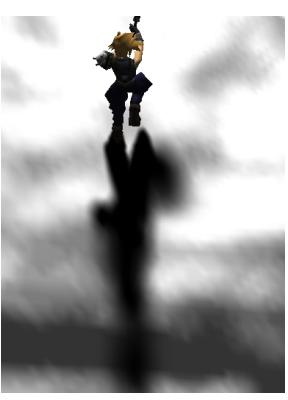

(c) Ground Truth

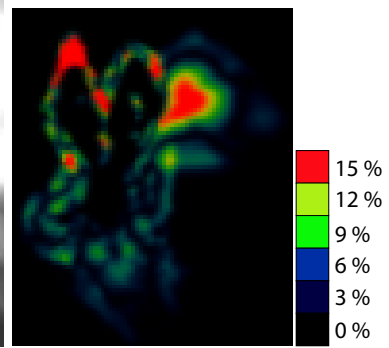

(d) Difference between the occlusion values

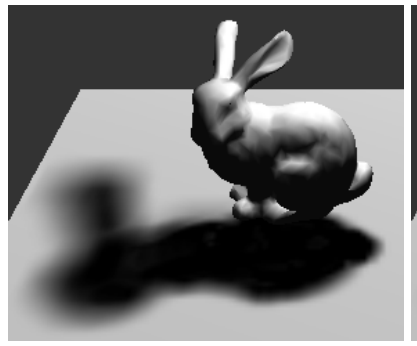

(e) Our algorithm

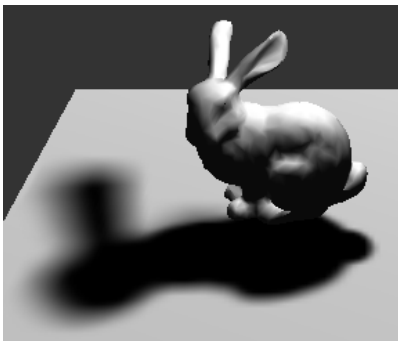

(f) Ground Truth

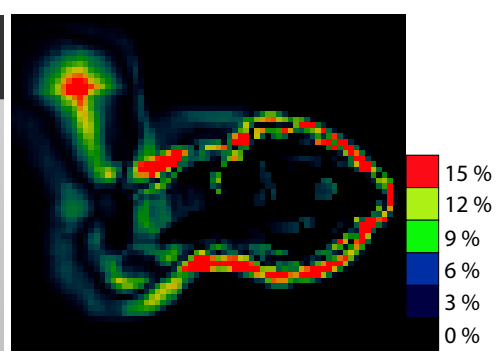

(g) Difference between the occlusion values

Figure 16: Visual comparison of our algorithm with ground truth.

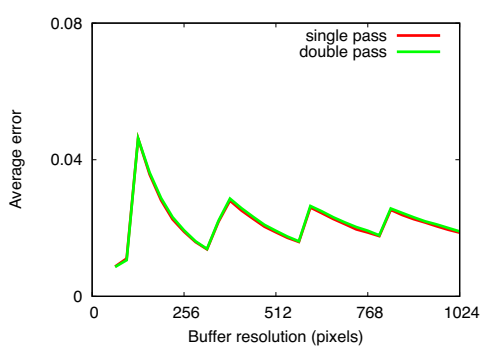

(a) Square plane

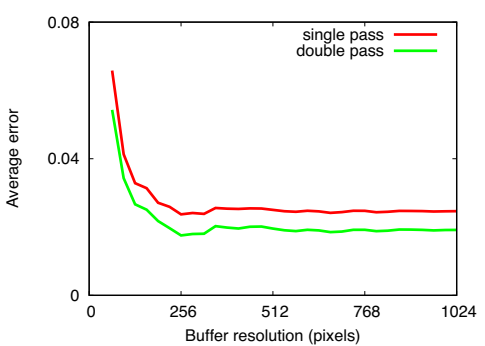

(b) Buddha

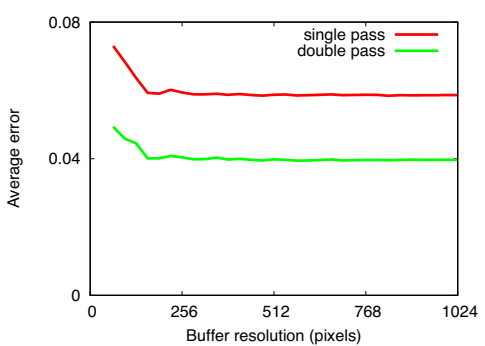

(c) Bunny

Figure 17: Variation of the error with respect to the resolution of the shadow map 
is the number of pixels for one side of the shadow map, so 128 corresponds to a $128 \times 128$ shadow map. For this test, we used non-power of two textures, in order to have enough sampling data. We can make several observations by looking at the data:

Two-pass version: the two-pass version of the algorithm consistently outperforms the single-pass version, always giving more accurate results. The only exception is of course the square plane: since it has no thickness, the single-pass and two-pass version give the same results.

Shadow map Resolution: as expected from the theoretical study (see Section 5.1.2), the error decreases as the resolution of the shadow map increases. What is interesting is that this effect reaches a limit quite rapidly. Roughly, increasing the shadow map resolution above 200 pixels does not bring an improvement in quality. Since the computation costs are related to the size of the shadow map, shadow map sizes of $200 \times 200$ pixels are close to optimal.

The fact that the error does not decrease continuously as we increase the resolution of the occluder map is a little surprising at first, but can be explained. It is linked to the silhouette effect. As we have seen in Fig. 16, the error introduced by our algorithm comes from the boundary of the silhouette of the occluder, from parts of the occluder that are not visible from the center of the light source, but visible from other parts of the light source. Increasing the resolution of the shadow map does not solve this problem. The optimal size for the shadow map is related to the size of the light source. As the light source gets larger, we can use smaller bitmaps.

Discretization error: the error curve for the square plane presents many important spikes. Looking at the results, it appears that these spikes correspond to discretization error (see Section 5.1.1). Since the square occluder is aligned with the axis of the shadow map, it magnifies discretization error.

\subsubsection{Size of the light source}

Figure 18 shows the average difference between the occlusion values computed with our algorithm and the actual occlusion values when we change the size of the light source for our three test scenes. The parameter values range from a point light source (parameter $=0.01$ ) to a very large light source, approximately as large as the occluder (parameter $=0.2$ ). We used a bitmap of $128 \times 128$ pixels for all these tests. We can make several observations by looking at the data:

Point light sources: the beginning of the curves (parameter=0.01) corresponds to a point light source. In that case, the error is quite large. This corresponds to an error of 1 , over the entire shadow boundary; as we are computing the shadow of the discretized occluder, we miss the actual shadow boundary, sometimes by as much as half a pixel. The result is a large error, but it occurs only at the shadow boundary.

Light source size: except for the special case of point light sources, the error increases with the size of the light source. This is consistent with our theoretical analysis (see Section 5.1.2).

\subsubsection{Occluder moving laterally}

Figure 19 shows the average difference between the occlusion values computed with our algorithm and the actual occlusion values, when we move the occluder from left to right under the light source. The parameter corresponds to the position with respect to the center of the light, with 0 meaning that the center of the object is aligned with the center of the light. We used a bitmap of $128 \times 128$ for all these tests.

The error is at its minimum when the occluder is roughly under the light source, and increases as the occluder moves laterally. The Buddha and Bunny models are not symmetric, so their curves are slightly asymmetric, and the minimum does not correspond exactly to 0 .

\subsubsection{Occluder moving vertically}

Figure 20 shows the average difference between the occlusion values computed with our algorithm and the actual occlusion values, when we move the occluder vertically. The smallest value of the parameter corresponds to an occluder touching the receiver, and the largest value corresponds to an occluder touching the light source. We used a bitmap of $128 \times 128$ for all these tests.

As predicted by the theory, the error increases as the occluder approaches the light source (see Section 5.1.2). For the Bunny, the error becomes quite large when the upper ear touches the light source.

\section{Complexity}

The main advantages of our algorithm are its rendering speed and its scalability. With a typical setup (a modern PC, an occluder map of $128 \times 128$ pixels, a scene between 50,000 polygons and 300,000 polygons), we get framerates between 30 and $150 \mathrm{fps}$. In this section, we study the numerical complexity of our algorithm and its rendering speed. We first conduct a theoretical analysis of the complexity of our algorithm, in Section 6.1, then an experimental analysis, where we test the variation of the rendering speed with respect to several parameters: the size of the shadow map, the number of polygons and the size of the light source (Section 6.2). Finally, in Section 6.3, we compare the complexity of our algorithm with a state-of-the-art algorithm, Soft Shadow Volume [AAM03].

\subsection{Theoretical complexity}

Our algorithm starts by rendering a shadow map and downloading it into main memory. This preliminary step has a 


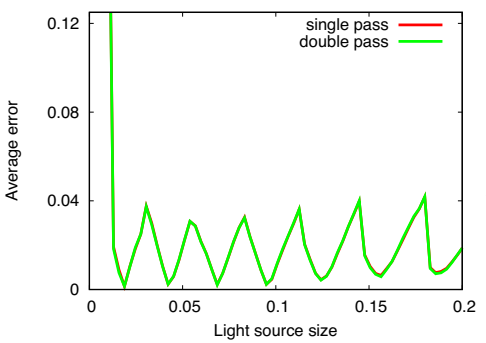

(a) Square plane

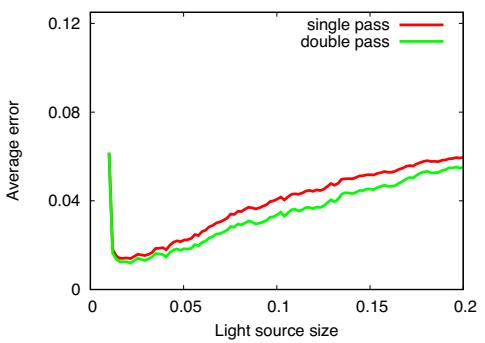

(b) Buddha

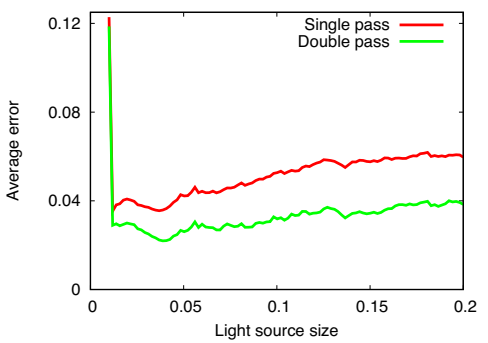

(c) Bunny

Figure 18: Variation of the error with respect to the size of the light source

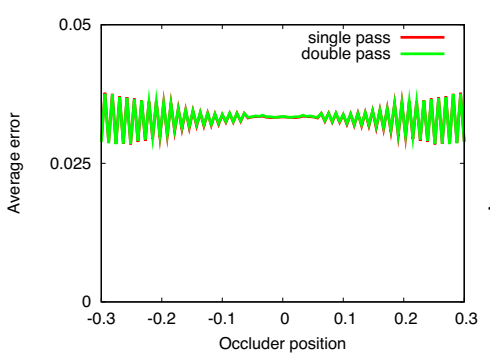

(a) Square plane

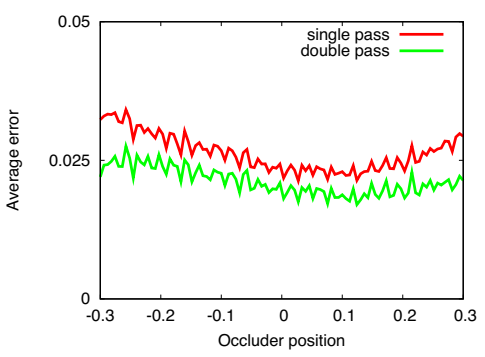

(b) Buddha

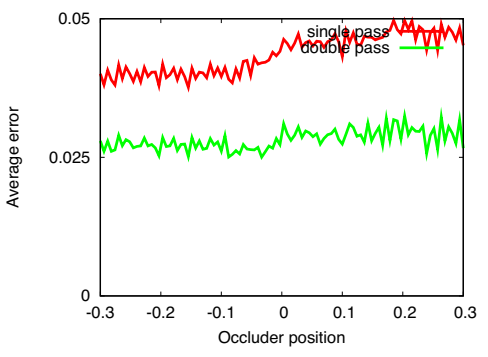

(c) Bunny

Figure 19: Variation of the error with respect to the lateral position of the occluder

complexity linear with respect to the number of polygons in the scene, and linear with the size of the shadow map, measured in the total number of pixels.

Then, for each pixel of the shadow map corresponding to the occluder, we compute its extent in the occlusion map, and for each pixel of this extent we execute a small fragment program of 11 instructions, including one texture lookup.

The overall complexity of this second step of the algorithm is the number of pixels covered by the occluder, multiplied by the number of pixels covered by the extent for each of them, multiplied by the cost of the fragment program. This second step is executed on the GPU, and benefits from the high-performance and the parallelism of the graphics card.

The worst case situation would be a case where each micro-patch in the shadow map covers a large number of pixels in the soft shadow map. But this situation corresponds to an object with a large penumbra zone, and if we have a large penumbra zone, we can use a lower resolution for the shadow maps. So we can compensate the cost for the algorithm by running it with bitmaps of lower resolution.

Using our algorithm with a large resolution shadow map in a situation of large penumbra results in relatively high computing costs, but a low resolution shadow map would give the same visual results, for a smaller computation time.

\subsection{Experimental complexity}

All measurements in this section were conducted on a 2.4 GHz Pentium4 PC with a GeForce 6800 Ultra graphics card. All framerates and rendering times correspond to observed framerates, that is the framerate for a user manipulating our system. We are therefore measuring the time it takes to display the scene and to compute soft shadows, not just the time it takes to compute soft shadows.

\subsubsection{Number of polygons}

We studied the influence of the polygon count. Fig. 6.2 shows the observed rendering time (in ms) as a function of the polygon count, with a constant occluder map size of $128 \times 128$ pixels. The first thing we note is the speed of our algorithm: even on a large scene of 340,000 polygons, we achieve real-time framerates (more than 30 frames per second). Second, we observe that the rendering time varies linearly with respect to the number of polygons. That was to be expected, as we must render the scene twice (once for the occluder map and once for the actual display), and the time it takes for the graphics card to display a scene varies linearly with respect to the number of polygons. For smaller scenes (less than 10,000 polygons, rendering time below 10 $\mathrm{ms}$ ), some factors other than the polygon count play a more important role.

Our algorithm exhibits good scaling, and can handle significantly large scenes without incurring a high performance 
L. Atty et al. / Soft Shadow Maps

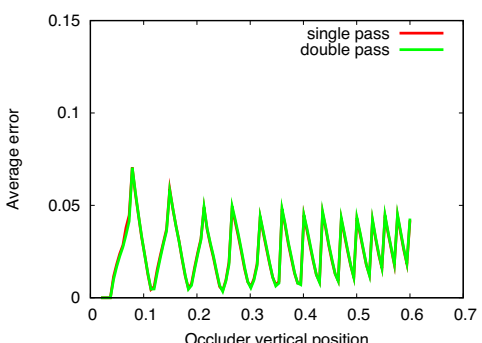

(a) Square plane

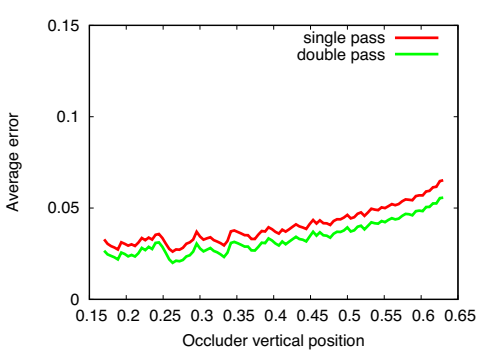

(b) Buddha

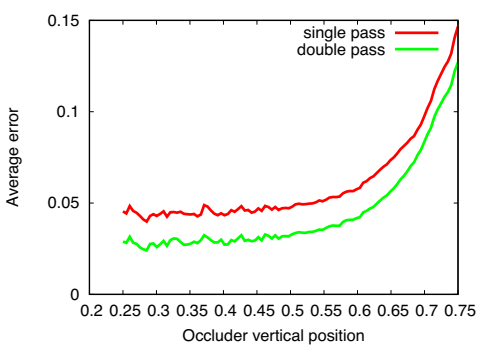

(c) Bunny

Figure 20: Variation of the error with respect to the vertical position of the occluder

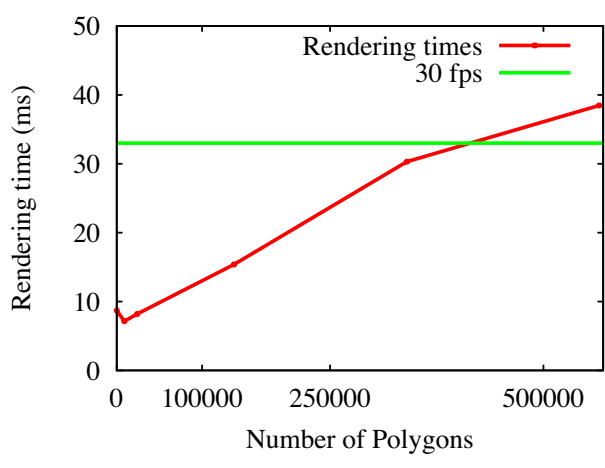

(a) Rendering times (in ms)

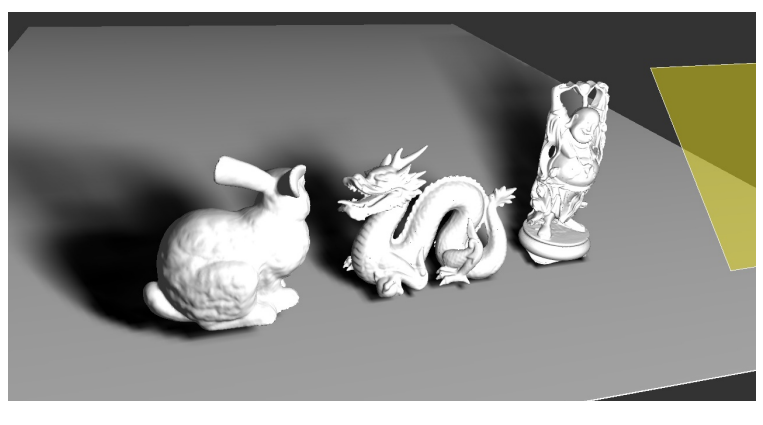

(b) Our largest test scene (565,203 polygons)

Figure 21: Influence of polygon count

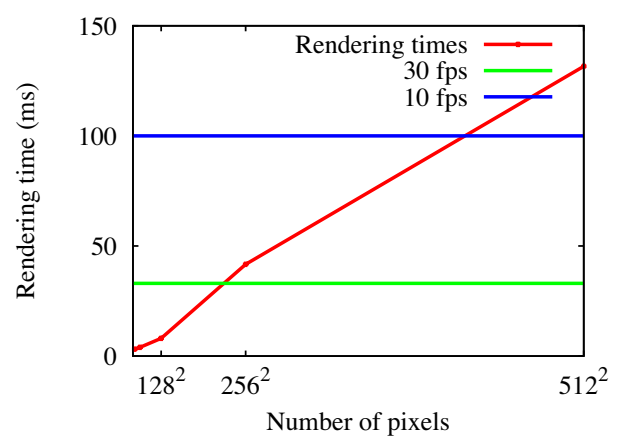

(a) Rendering times (in $\mathrm{ms}$ )

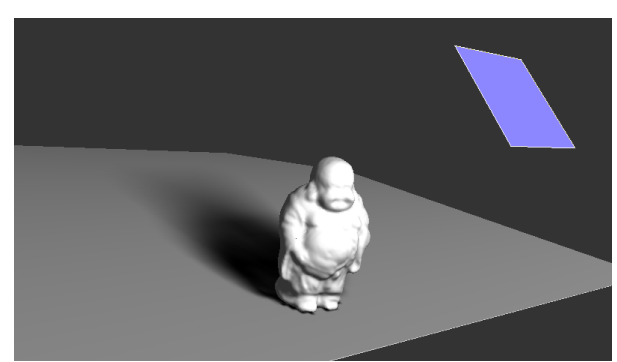

(b) Test scene (24,000 polygons)

Figure 22: Influence of the size of the occluder map 


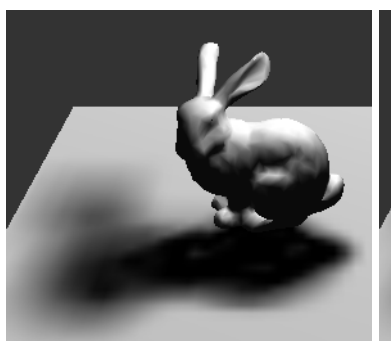

(a) Bitmap of $64^{2}$ (184 fps)

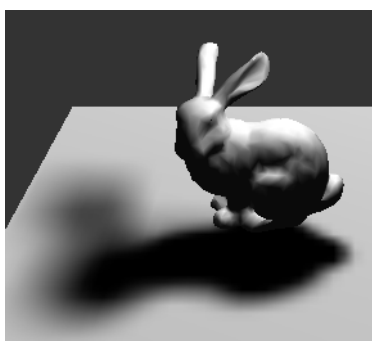

(b) Ground truth
Figure 23: Large light sources with small bitmaps

cost. The maximum size of the scene depends on the requirements of the user.

\subsubsection{Size of occluder map}

Fig. 6.2.1 shows the observed rendering times (in $\mathrm{ms}$ ) of our algorithm, on a scene with 24,000 polygons (Fig. 22(b)), when the size of the occluder map changes. We plotted the rendering time as a function of the number of pixels in the occluder map (that is, the square of the size of the occluder map) to illustrate the observed linear variation of rendering time with respect to the total number of pixels.

An occluder map of $512^{2}$ pixels gives a rendering time of $150 \mathrm{~ms}$ - or $7 \mathrm{fps}$, too slow for interactive rendering. An occluder map of $128^{2}$ or $256^{2}$ pixels gives a rendering time of 10 to $50 \mathrm{~ms}$, or 20 to $100 \mathrm{fps}$, fast enough for real-time rendering. For a large penumbra region, an occluder map of $128^{2}$ pixels qualitatively gives a reasonable approximation, as in Fig. 22(b). For a small penumbra region, our algorithm behaves like the classical shadow mapping algorithm and artifacts can appear with a small occluder map of $128^{2}$ pixels; in that case, it is better to use $256^{2}$ pixels.

The fact that the rendering time of our algorithm is proportional to the number of pixels in the occluder map confirms that the bottleneck of our algorithm is its transfer to the CPU. Due to the cost of this transfer, we found that for some scenes it was actually faster to use textures whose dimensions are not a power of 2: if the difference in pixel count is sufficient, the gain in transfer time compensates the losses in rendering time.

\subsubsection{Light source size}

Another important parameter is the size of the light source, compared to the size of the scene itself. A large light source results in a large penumbra region for each micro-patch, resulting in more pixels of the soft shadow map covered, and a larger computational cost. Fig. 24(a) shows the observed framerate as a function of the size of the light source. We did the tests with several bitmap resolutions $\left(256^{2}, 128^{2}, 64^{2}\right)$. Fig. 24(b) shows the error as a function of the size of the light source, for the same bitmap resolutions.
As you can see from Fig. 24(a), the rendering time increases with the size of the light source. What is interesting is the error introduced by our algorithm (see Fig. 24(b)). The error logically increases with the size of the light source, and for small light sources, larger bitmaps result in more accurate images. But for large light sources, a smaller bitmap will give a soft shadow of similar quality. A visual comparison of the soft shadows with a small bitmap and ground truth shows the small bitmap gives a very acceptable soft shadow (see Fig. 23).

This effect was observed by previous researchers: as the light source becomes larger, the features in the soft shadow become blurrier, hence they can be modeled accurately with a smaller bitmap.

\subsection{Comparison with Soft-Shadow Volumes}

Finally, we performed a comparison with a state-of-the art algorithm for computing soft shadows, the Soft-Shadow Volumes by Assarsson and Akenine-Möller [AAM03].

Fig. 25 shows the same scene, with soft shadows, computed by both algorithms. We ran the tests with a varying number of jeeps, to test how both algorithms scale with respect to the number of polygons. Fig. 25(c) shows the rendering times as a function of the number of polygons for both algorithms. These figures were computed using a window of $512 \times 512$ pixels for both algorithms, and with the two-pass version of our algorithm, with an occluder map resolution of $210 \times 210$.

Our algorithm scales better with respect to the number of polygons. On the other hand, soft shadow volumes provide a better looking shadow (see Fig. 25(b)), closer to the actual truth.

It is important to remember that the rendering time for the Soft- Shadow Volumes algorithm varies with the number of screen pixels covered by the penumbra region. If the viewpoint is close to a large penumbra region, the rendering time becomes much larger. The figures we used for this comparison correspond to an observer walking around the scene (as in Fig. 25(b)).

\section{Conclusion and Future Directions}

In this paper, we have presented a new algorithm for computing soft shadows in real-time on dynamic scenes. Our algorithm is based on the shadow mapping algorithm, and is entirely image-based. As such, it benefits from the advantages of image-based algorithms, especially speed.

The largest advantage of our algorithm is its high framerate, hence there remains plenty of computational power available for performing other tasks, such as interacting with the user or performing non-graphics processing such as physics computations within game engines. Possibly the 


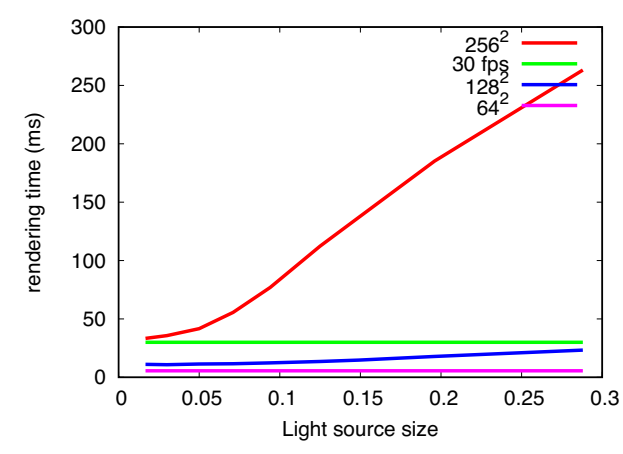

(a) Rendering time

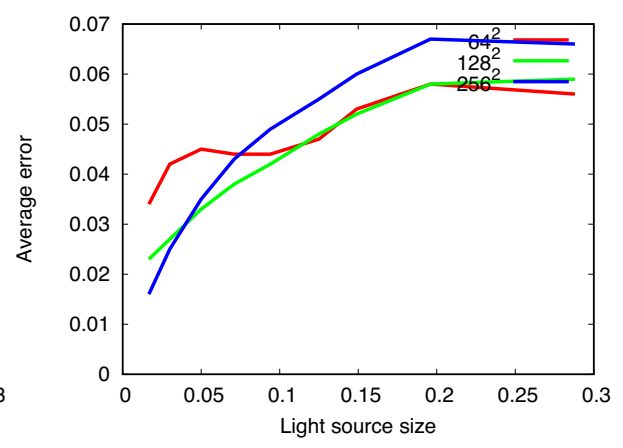

(b) Average error

Figure 24: Changing the size of the light source (floating bunny scene)

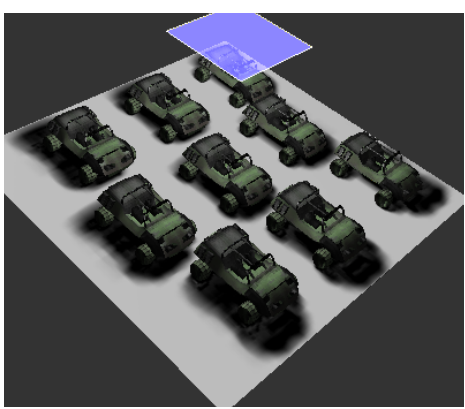

(a) Soft Shadow Maps

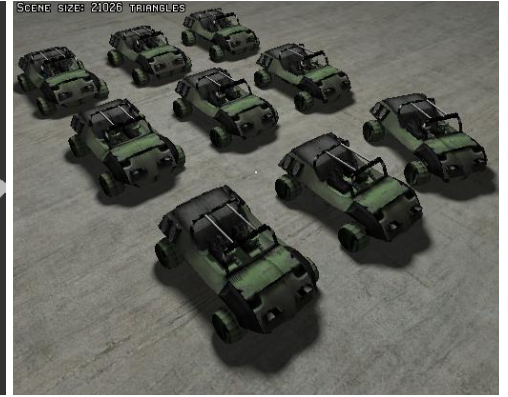

(b) Soft Shadow Volumes

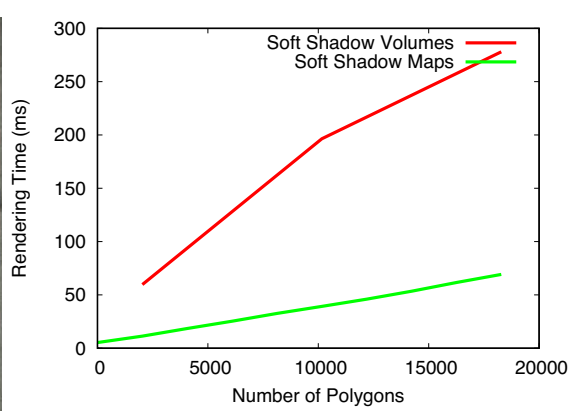

(c) Rendering times

Figure 25: Comparison with Soft-Shadow Volumes

largest limitation of our algorithm is the fact that it does not compute self-occlusion and it requires a separation between occluders and receivers. We know that this limitation is very important, and we plan to remove it in future work, possibly by using layered depth images.

An important aspect of our algorithm is that we can use low-resolution shadow maps in places with a large penumbra, even though we still need higher resolution shadow maps for places with small penumbra, for example close to the contact between the occluder and the receiver. An obvious improvement to our algorithm would be the ability to use hierarchical shadow maps, switching resolutions depending on the shadow being computed. This work could also be combined with perspective-corrected shadow maps [SD02, WSP04, MT04, CG04], in order to have higher resolution in places with sharp shadows close to the viewpoint.

In its current form, our algorithm still requires a transfer of the occluder map from the GPU to the main memory, and a loop, on the CPU, over all the pixels in the occluder map. We would like to design a GPU only implementation of our algorithm, using the future render-to-vertex-buffer capabilities.

\section{Acknowledgments}

Most of this work was conducted while Charles Hansen was on sabbatical leave from the University of Utah, and was a visiting professor at ARTIS/GRAVIR IMAG, partly funded by INPG and INRIA.

The authors gratefully thank Ulf Assarsson for doing the tests for the comparison with the soft-shadow volumes algorithm.

The Stanford Bunny, Happy Buddha and dragon 3D models appearing in this paper and in the accompanying video were digitized and kindly provided by the Stanford University Computer Graphics Laboratory.

The smaller Buddha 3D model appearing in this paper was digitized and kindly provided by Inspeck.

The Jeep 3D model appearing in this paper was designed and kindly provided by Psionic.

The horse 3D model appearing in the accompanying video was digitized by Cyberware, Inc., and was kindly provided by the Georgia Tech. "Large Geometric Models Archive".

The skeleton foot 3D model appearing in the accompanying video was digitized and kindly provided by Viewpoint Datalabs Intl.

\section{References}

[AAM03] Assarsson U., AKenine-Möller T.: A geometry-based soft shadow volume algorithm using 
graphics hardware. ACM Transactions on Graphics (Proc. of SIGGRAPH 2003) 22, 3 (2003), 511-520.

[AHT04] ARVo J., HiRvikorPI M., TyYstJärVi J.: Approximate soft shadows using image-space flood-fill algorithm. Computer Graphics Forum (Proc. of Eurographics 2004) 23, 3 (2004), 271-280.

[AMH02] Akenine-Möller T., Haines E.: Real-Time Rendering, 2nd ed. A. K. Peters, 2002.

[BS02] BRABeC S., Seidel H.-P.: Single sample soft shadows using depth maps. In Graphics Interface (2002).

[CD03] ChAn E., DuRAND F.: Rendering fake soft shadows with smoothies. In Rendering Techniques 2003 (Proc. of the Eurographics Symposium on Rendering) (2003), pp. 208-218.

[CD04] Chan E., Durand F.: An efficient hybrid shadow rendering algorithm. In Rendering Techniques 2004 (Proc. of the Eurographics Symposium on Rendering) (2004), pp. 185-195.

[CG04] Chong H., Gortler S. J.: A lixel for every pixel. In Rendering Techniques 2004 (Proc. of the Eurographics Symposium on Rendering) (2004), pp. 167-172.

[Cro77] CROw F. C.: Shadow algorithms for computer graphics. Computer Graphics (Proc. of SIGGRAPH '77) 11, 2 (1977), 242-248.

[DF94] Drettakis G., Fiume E.: A fast shadow algorithm for area light sources using backprojection. In $S I G$ GRAPH '94 (1994), pp. 223-230.

[GBP06] Guennebaud G., Barthe L., Paulin M.: Real-time soft shadow mapping by backprojection. In Rendering Techniques 2006 (Proc. of the Eurographics Symposium on Rendering) (2006).

[HLHS03] Hasenfratz J.-M., LAPIERRE M., Holzschuch N., Sillion F.: A survey of realtime soft shadows algorithms. Computer Graphics Forum 22, 4 (2003), 753-774.

[KD03] KiRSCH F., DOELlner J.: Real-time soft shadows using a single light sample. Journal of WSCG (Winter School on Computer Graphics) 11, 1 (2003).

[KMK97] Kersten D., Mamassian P., Knill D. C.: Moving cast shadows and the perception of relative depth. Perception 26, 2 (1997), 171-192.

[LWGM04] Lloyd B., Wendt J., Govindaraju N., MANOCHA D.: Cc shadow volumes. In Rendering Techniques 2004 (Proc. of the Eurographics Symposium on Rendering) (2004), pp. 197-205.

[McC00] McCool M. D.: Shadow volume reconstruction from depth maps. ACM Transactions on Graphics 19, 1 (2000), 1-26.

[MT04] MARTIN T., TAN T.-S.: Anti-aliasing and continuity with trapezoidal shadow maps. In Rendering Tech- niques 2004 (Proc. of the Eurographics Symposium on Rendering) (2004), pp. 153-160.

[SD02] Stamminger M., Drettakis G.: Perspective shadow maps. ACM Transactions on Graphics (Proc. of SIGGRAPH 2002) 21, 3 (2002), 557-562.

[SS98] Soler C., Sillion F. X.: Fast calculation of soft shadow textures using convolution. In SIGGRAPH '98 (1998), pp. 321-332.

[Wan92] WANGer L.: The effect of shadow quality on the perception of spatial relationships in computer generated imagery. In Symposium on Interactive 3D Graphics (1992), pp. 39-42.

[WFG92] Wanger L., Ferwerda J. A., GreenberG D. P.: Perceiving spatial relationships in computergenerated images. IEEE Computer Graphics and Applications 12, 3 (1992), 44-58.

[Wh03] Wyman C., Hansen C.: Penumbra maps: Approximate soft shadows in real-time. In Rendering Techniques 2003 (Proc. of the Eurographics Symposium on Rendering) (2003), pp. 202-207.

[Wil78] Williams L.: Casting curved shadows on curved surfaces. Computer Graphics (Proc. of SIGGRAPH '78) 12, 3 (1978), 270-274.

[WPF90] Woo A., Poulin P., Fournier A.: A survey of shadow algorithms. IEEE Computer Graphics \& Applications 10, 6 (1990), 13-32.

[WSP04] Wimmer M., ScherZer D., Purgathofer W.: Light space perspective shadow maps. In Rendering Techniques 2004 (Proc. of the Eurographics Symposium on Rendering) (2004), pp. 143-152.

Appendix A: Floating-point blending accuracy

In this section, we review the issues behind the hardware blending accuracy problems we have encountered and propose a temporary fix for these issues.

All the accuracy issues are linked to the fact that hardware blending is, at the time of writing, only available for 16-bits floating point numbers. NVidia graphics hardware stores these floating-point numbers using s10e5 format: one bit of sign, 10 bits of mantissa, 5 bits of exponent, with a bias of 15 for the exponent. The important point for addition is that the mantissa is stored on 10 bits. As a result, adding a large number $X$ and a small number $\varepsilon$ will give an inaccurate result if $\varepsilon<2^{-10} X$ :

$$
X+\varepsilon=X \quad \text { if } \varepsilon<2^{-10} X(\text { inFP16) }
$$

For example, $2048+1=2048$ (in FP16 format) and $0.5+$ $\frac{1}{2049}=0.5$ (also in FP16 format).

In some cases, the addition of the contribution from all micro-patches will be 1 (meaning complete occlusion of the light source). As a consequence, we can expect numerical 
accuracy issues if some micro-patches hide less than $2^{-10}$ of the light source. Because $32^{2}=2^{10}$, it means that the width of the reprojection of one micro-patch should be larger than $\frac{1}{32}$ of the width of the light source.

This translates easily into conditions for the position of the occluder:

$$
\frac{1}{z_{O}}<\frac{1}{z_{R}}+\frac{64 \tan \alpha}{N L}
$$

where $L$ is the width of the light source, $N$ is the resolution of the bitmap, $\alpha$ is the half-angle of the camera used to generate the shadow map, $z_{O}$ is the distance between the light source and the occluder and $z_{R}$ is the distance between the light source and the receiver.

Bitmap resolution: The most important thing is that increasing $N$ makes this error more likely to appear. This explains why using a bitmap of $512 \times 512$ pixels we see a poor looking shadow, while the $128 \times 128$ bitmap gives the correct shadow (see Fig. 14).

Light source size: In equation 4, the size of the light source appears in a product with the resolution of the bitmap. If the light source is large, the bitmap must be low resolution in order to avoid FP16 blending errors. Fortunately, a large light source means a large penumbra for most occluders, so a low resolution bitmap might be enough for these penumbra effects.

Occluder position: As the occluder moves closer to the receiver, the likeliness of blending errors gets lower.

Camera half-angle: Similarly, increasing the camera halfangle improves the FP16 blending accuracy.

Basically, all these conditions amount to the same thing: using less pixels to describe the occluder in the shadow map. While this improves the FP16 blending accuracy, it obviously degrades the discretization of the occluder and also increases the overlapping between reprojections of neighboring pixels.

In our experiments (see Fig. 14) the blending accuracy problem appears very often when the resolution of the shadow map is larger than $512 \times 512$, sometimes with a shadow map resolution of $256 \times 256$ and very rarely with a shadow map resolution of $128 \times 128$.

The problem will disappear when hardware blending will become available on higher accuracy floating point numbers. FP32 have a mantissa of 23 bits, allowing the use of micropatches that block less than $2^{-23}$ of the light source, meaning that the width of the back-projection of the micro-patch should be at least larger that $2^{-11}$ than the width of the light source (64 times smaller than the current threshold). Compared with the current method, it would allow the use of shadow maps with a resolution above $4096 \times 4096$.

With FP16 blending only, the best solution is to use a hierarchical shadow map for soft-shadow computations, as was suggested by Guennebaud et al. [GBP06]: the low resolution shadow map would be used for large penumbra regions, and the high-resolution shadow map for areas with hard shadows, e.g. when the occluder and the receiver are in contact. 